\title{
Acessando o medo do crime: Um survey por meio da internet
}

\author{
Accessing the fear of crime: An internet survey
}

Carlos Eduardo Pimentel ${ }^{[a]}$, Hartmut Gunther ${ }^{[b]}$ Peter Ulrich Vieth Black ${ }^{[c]}$

\footnotetext{
${ }^{[a]}$ Bolsista de Doutorado do CNPq, Programa de Pós-Graduação em Psicologia Social, do Trabalho e das Organizações, Brasília, DF - Brasil, e-mail: carlospimentel@unb.br; carlosepimentel@bol.com.br.

${ }^{[b]}$ PhD em Psicologia Social, professor titular da Universidade de Brasília (UnB), Brasília, DF - Brasil, e-mail: hartmut.gunther@gmail.com

${ }^{[c]}$ Aluno de mestrado no Programa de Pós-Graduação em Psicologia Social, do Trabalho e das Organizações da Universidade de Brasília, (UnB), Brasília, DF - Brasil, e-mail: peter.uvb@gmail.com
}

Recebido: 28/03/2011 Received: 03/28/2011

Aprovado: $17 / 05 / 2011$ Approved: 05/17/2011

\section{Resumo}

É reconhecida a importância dos meios de comunicação de massa nos processos de socialização e influência. Todos os dias somos informados pelos meios de comunicação de massa, principalmente pela TV, de uma grande quantidade de crimes. A transmissão de tais comportamentos pode suscitar medo na audiência, mas também ajudá-los a se proteger. Com o objetivo de conhecer variáveis correlacionadas com esse medo, realizou-se um survey pela internet na cidade de Brasília, por meio da tradução da Escala do Medo do Crime que foi utilizada nos EUA, com o objetivo de verificar possíveis relações desse medo com a exposição à TV, gênero e idade com base na teoria do cultivo e hipótese da vulnerabilidade. Os principais resultados apoiam a teoria do cultivo, mas não se verificou relação do medo do crime com o gênero e a idade dos participantes. Todavia talvez as relações não sejam simplesmente lineares. Os principais resultados são discutidos fundamentadas na literatura especializada e sugestões de estudos futuros foram realizadas.

Palavras-chave: Medo do crime. Horas semanais de TV. Gênero e idade.

\section{Abstract}

The importance of mass media in the socialization and influence processes has been much recognized. Everyday we are informed by these media, especially by the television, about plenty of crimes. Broadcasting such behaviors may generate fear in the audience, but may also help them protect themselves. With the aim of searching variables correlated with this fear, a Web Survey using a translated Fear of Crime Scale - which is in use in the U.S.A. - was carried out to ascertain the relation of TV exposure, gender and age based on the cultivation theory and on the vulnerability hypothesis. The main results support the cultivation theory, but there was no relation identifiable among fear of crime, age and gender of participants. However, maybe those relations are not simply linear. The main results are discussed based on specialized literature and suggestions for future studies were made.

Keywords: Fear of crime. Weekly hours before TV. Gender and age. 
Jack é ágil, Jack é rápido Assalta seu bolso, transforma em trapaça Devagar e constante, ele tem tempo Para cometer outro crime de TV Crime de TV

(Black Sabbath - TV Crimes, 1992)

A cada dia que passa assistimos a mais e mais comportamentos criminais mediados pela programação televisiva. Cotidianamente ficamos perplexos com a quantidade de delitos, crimes de violência extrema; homicídios, latrocínios, sequestros, ataques a policiais, confrontos armados que são transmitidos. Muito além dos antigos filmes do velho oeste ou dos contemporâneos longas de ação, policiais, cenas da realidade social como o confronto de traficantes e policiais, balas perdidas ou aquelas com alvo escolhido, fazem do dia a dia um verdadeiro temor. É recorrente à metáfora que os verdadeiros presos estão dentro de casa, acuados pelo medo do crime, protegidos pela segurança de grades, portas e cães de guarda. Neste sentido, o medo do crime se converte num tema de grande importância para as Ciências Sociais e de modo especial para a Psicologia, dado que tem uma influência paralisante no comportamento. Evidentemente, este é um problema que atravessa as fronteiras do nosso país.

Em termos mundiais, o Brasil se encontra em sexto lugar - ficando atrás apenas de El Salvador, Colômbia, Guatemala, I. Virgens (EUA) e Venezuela, no que diz respeito às taxas de homicídios na população total. Tais dados mostram um quadro muito preocupante para a América Latina quanto ao ranking internacional da violência. Mais precisamente para o caso brasileiro, trata-se de uma taxa de 25,8 homicídios por 100 mil habitantes (Waiselfisz, 2010). Esta edição do Mapa da violência também mostrou que num período de dez anos, considerando de 1997 a 2007, o número total de homicídios no Brasil foi de 40.507 a 47.707 , representando um aumento de quase $18 \%$ em todas as regiões do país. Considerando a capital, em Brasília houve uma redução nas taxas de homicídios de 1997 para 2007 de 35,6 (13a posição em relação às capitais brasileiras) para 33,5 (17a posição) em 100.000 na população total.

Todavia quando se considera o Distrito Federal (DF), estado no qual se realizou a presente pesquisa, verifica-se que a situação das taxas de homicídio, considerando os anos de 1997 (35,5) e 2007 (33,5), é algo preocupante, ocupando a sexta e a quinta posições no ordenamento das 27 Unidades Federativas. Além disso, quando se considera apenas a população de 0 a 19 anos, verifica-se que o DF passa a ocupar a segunda $(19,7)$ e a quarta $(24,2)$ posições nas taxas de homicídio em termos de Brasil nos anos de 1997 e 2007. Considerando agora a população de 15 a 24 anos, verificamos um total de 3.768 homicídios de 1997 a 2007. Ademais, o percentual de homicídios do DF sempre esteve acima do percentual do Centro-Oeste nesse período de anos (Waiselfisz, 2010).

\section{Medo do crime e teoria do cultivo}

O medo do crime pode ser entendido como um indicador das preocupações com a segurança (Gorman-Smith, Tolan \& Henry, 2000). Este construto pode ainda ser entendido como medos, sentimentos, julgamentos cognitivos ou emocionais e aspectos comportamentais relacionados ao crime (McCrea, Shyy, Western \& Stimson, 2005). Tem-se argumentado, ademais, que, mesmo que se tenha tratado comumente o medo do crime como uma resposta emocional perante o crime, este tem sido mais pesquisado a partir de atitudes e crenças (Mora, 2009). Conforme explica o autor, tal medo tem se baseado em atitudes face à preocupação com o crime, julgamentos probabilísticos sobre ser vítima ou mesmo a raiva.

De acordo com a literatura especializada, os principais atributos que predizem o medo do crime são o sexo e a idade, dando apoio empírico à hipótese da vulnerabilidade (Barnett, Buys, Lovie-Kitchin, Boulton-Lewis, Smith \& BBus, 2007; McCrea et al., 2005; Fetchenhauer \& Buunk, 2005). Esta hipótese sugere que as mulheres e pessoas idosas são as que mais sofrem medo do crime, pois são mais fisicamente vulneráveis.

Existem, por outro lado, evidências substanciais que assistir à cobertura do crime pela televisão tem se relacionado com mais medo do crime (Gerbner \& Gross, 1976; Holbert, Shah \& Kwak, 2004; O’Keefe \& Reid-Nash, 1987; Romer, Jamieson \& Aday, 2003). A propósito, desde a popularização da TV nos Estados Unidos e Inglaterra durante os anos 1950, o impacto deste meio de comunicação de massas tem capturado a atenção das Ciências Sociais, convertendo-se em tema de intensivo debate, principalmente pelas altas taxas de violência noticiadas (Gunter \& Harrison, 2005). Gerbner e Gross (1976) explicaram o poder 
da televisão como meio de socialização, formação e padronização de papéis, comportamentos, opiniões e concepções da realidade social; processo chamado de enculturação. No âmbito da Psicologia Social, a influência dos meios de comunicação de massa (como filmes documentários) tem sido testada, com estudos do efeito desses meios na mudança de atitudes (Johnson, Maio \& Smith-McLallen, 2005; Weiss, 1968).

A teoria da cultivação ou do cultivo desenvolvida por George Gerbner e seus colaboradores (Gerbner, 1970; Gerbner \& Gross, 1976; Gerbner, Gross, Morgan \& Signorielli,1980a) tratou, não exclusivamente mas especialmente, da influência da violência televisionada (DeFleur \& Ball-Rokeach, 1993; Gerbner, 1970; Gerbner \& Gross, 1976; Gerbner et al., 1980a; Rangel, 2004). A análise da cultivação foi definida por seus autores como sendo as concepções cultivadas nos telespectadores acerca da realidade social relacionadas aos aspectos mais salientados desta realidade pela televisão (Gerbner et al., 1980a). Ou seja, Gerbner (1970) tratou do estudo da mensagem dirigida para as massas e o estudo das formas, funções e processos de tomada de decisões que configuram este sistema de mensagens. Definiu a análise do cultivo como "o estudo das relações entre processos institucionais, sistemas de mensagens e pressupostos públicos, imagens e políticas que cultivam" (Gerbner, 1970, p. 71). Gerbner e seus colaboradores realizaram análises de conteúdo da programação televisiva, o projeto de Indicadores Culturais, por tipo de violência (considerando-se a expressão direta de força física com objetivo de matar ou ferir) durante pelo menos 20 anos (Gunter \& Harrison, 2005).

Dita análise, que encontrou um alto percentual de violência consistente ao longo dos anos, chegando até a 27 atos de violência por hora, indicou um retrato não acurado da realidade (Bryant \& Miron, 2004) e a conclusão de Gerbner e equipe não foi novidade: a realidade apresentada pela TV pode influenciar crenças e comportamentos (DeFleur \& Ball-Rokeach, 1993). Mais contemporaneamente, ao se comparar com dados disponibilizados pelo U.S. Federal Bureau of Investigation (FBI), verificou-se que a violência em programas de TV, baseados na realidade, foi bem maior do que aqueles verificados na vida real (Bushman \& Anderson, 2001).

A teoria em apreço, das mais utilizadas na pesquisa em comunicação de massas (Bryant \& Miron, 2004; Saito, 2007), é particularmente útil para se entender o medo do crime; de vez que prediz que, quanto mais as pessoas assistem à violência televisionada, mais medo têm do crime no mundo real. Mas têm sido utilizadas para se entender outros temas divulgados na televisão também, como crenças relacionadas ao câncer, atitudes diante do gênero (Niederdeppe, Fowler, Goldstein \& Pribble, 2010; Saito, 2007) e também para os efeitos de se jogar vídeo games online violentos (Williams, 2006).

Gerbner e Gross (1976, p. 190) verificaram no seu survey que a programação televisiva influenciou concepções acerca da realidade social e destacaram: "temos indícios de que assistir à televisão cultiva um sentido geral de perigo e desconfiança". Os autores compararam respostas de adultos que despendiam muitas (uma média de quatro horas diárias ou mais) e poucas horas assistindo à TV (uma média de duas horas diárias ou menos) e verificaram que $52 \%$ dos que mais assistiam TV acreditaram ter mais chances de serem vítimas de violência, contra 39\% dos que assistiam menos (Gerbner \& Gross, 1976). Crenças sobre o crime na realidade foram cultivadas pela programação televisiva, que mostrou quantidades de crimes ao telespectador, levando-os à crença no mundo perigoso (DeFleur \& Ball-Rokeach, 1993).

Neste artigo (Gerbner \& Gross, 1976), não existem informações a respeito de que modo foi realizado o survey. Os autores remetem a um relatório para quem quiser mais detalhes com relação ao método. No entanto, verifica-se que Gerbner, Gross, Morgan e Signorielli (1984) realizaram survey utilizando-se de questionários autoadministráveis em sala de aula. Ademais, de acordo com Gerbner et al. (1984), amplos levantamentos que Gerbner e seus colaboradores realizaram nos anos de 1975, 1977, 1978 e 1982, utilizaram amostras probabilísticas, por quotas e longas entrevistas pessoais.

Com o objetivo de verificar se o medo do crime estava mais presente entre aqueles que assistiam mais à TV, Doob e McDonald (1979) planejaram um survey domiciliar, na cidade de Toronto, no Canadá, em áreas de alta e baixa prevalência de crime. Doob e McDonald encontraram que aqueles respondentes que assistiam mais à TV (considerando quantidade total de TV) reportaram mais medo do crime com a amostra total $(r=0,18, p<0,001)$ e especificamente nas áreas de alto crime $(r=0,24, p<0,05)$. Esse padrão de correlação se repetiu quando se correlacionou o total de TV violenta com o medo do crime com 
a amostra total ( $r=0,18, p<0,001)$ e especificamente nas áreas de alto crime $(r=0,22, p<0,05)$, mas tais efeitos desaparecem nas áreas de baixa prevalência de crime. Os autores concluem que os resultados de Gerbner e colaboradores foram replicados. Adicionalmente, O'Keefe e Reid-Nash (1987) verificaram que assistir a notícias na TV sobre o crime se correlacionou positivamente com o medo de caminhar sozinho à noite, mas também com o medo de ser roubado e com preocupações com a segurança pessoal.

Ainda buscando verificar se o medo do crime poderia ser entendido como um produto da exposição a noticiários a respeito de crimes transmitidos pela TV, Romer et al. (2003) utilizaram dados de um survey realizado nos Estados Unidos de 1990 a 1995. De acordo com as análises os autores, o medo do crime se relacionou com assistir a notícias de crime na TV local, o que deu apoio empírico à teoria do cultivo. Por outro lado, Holbert et al. (2004) conduziram um survey por correio e verificaram que assistir a reality shows policiais predisse o medo do crime em $1999(\beta=0,12$, $\mathrm{p}<0,001)$ e $2000(\beta=0,09, \mathrm{p}<0,001)$, assim como noticiários sobre o crime: $(1999, \beta=0,04, p<0,05)$ e em $2000(\beta=0,06, p<0,01)$.

Recentemente foram testadas três explicações da relação entre assistir a TV e medo do crime: hipótese de cultivo (assistir a TV $\rightarrow$ medo do crime), whithdrawal (as pessoas que têm mais medo ficam mais em casa e assistem mais a TV) e manejo do humor (as pessoas que têm mais medo escolhem programas com mais crime para aprenderem a lidar com o medo), Bulck (2004) encontrou evidências favoráveis de que assistir a TV prediz o medo do crime por meio de equação estrutural testando modelos alternativos: g.l. $=85 ; \chi^{2}=169,4$; AGFI $=0,98$; $\mathrm{ECVI}=0,42$; RMSEA $=0,042$; $\mathrm{CAIC}=426,77$. Num outro survey realizado por telefone usando uma amostra de 1005 adultos, corroborou-se que assistir a crimes na TV predisse o medo do crime $(\beta=0,08$, $p<0,01$ ), controlando-se diversas variáveis sociodemográficas (como sexo, idade e renda), mas as horas de TV por semana não predisseram o medo do crime (Dowler, 2003). Nestes termos, é importante ressaltar que variáveis sócio demográficas têm sido analisadas com os efeitos do cultivo. Gerbner et al. (1980a), por exemplo, verificaram que os efeitos do cultivo no medo do crime foram maiores naqueles com maior renda e em brancos em comparação àqueles com menor renda e não brancos.

Mais recentemente, esta teoria foi testada no que diz respeito à influência da TV nas atitudes em relação ao gênero, sendo que a hipótese de "mainstreaming"; ou seja, de que a televisão produziria uma visão homogênea do mundo naqueles que mais assistem a TV não foi corroborada (Saito, 2007). Verificou-se, por outro lado, que a exposição a noticiários televisivos cultivou crenças acerca da prevenção ligada ao câncer na audiência, mas que não pode ser entendida como relações de causa e efeito (Niederdeppe, Fowler, Goldstein \& Pribble, 2010). Foi verificado também, que a partir de estudo experimental de campo, os efeitos de cultivação de se jogar vídeo games online violentos sugerem efeitos de dias e semanas nas percepções relacionadas ao crime (Williams, 2006).

Em uma meta-análise, verificou-se um coeficiente de correlação pequeno para os efeitos do cultivo (Morgan \& Shanahan, 1996), mas não significativo quando se controlavam variáveis demográficas. Porém Gerbner e colaboradores argumentaram que pequenas mudanças em variáveis podem levar a importantes consequências (Gerbner, Gross, Morgan, Signorielli \& Shanahan, 2002) e que suas correlações são pequenas, "mas estatisticamente significativas e consistentes em amostras de crianças, adolescentes e adultos" (Gerbner, Gross, Morgan \& Signorielli, 1979). Em suma, a teoria de Gerbner e colaboradores (Gerbner \& Gross, 1976, Gerbner et al., 1980ab) tem recebido apoio empírico, mas também tem sido criticada (Hirsch, 1980; Shrum, Burroughs \& Rindfleisch, 2004) incluindo reanálises com controles estatísticos que não comprovaram as predições da teoria (ver Hughes, $1980^{1}$; Wåhlberg \& Sjöberg, 2000).

Existe, teoricamente, maior probabilidade de que a audiência mais exposta à TV construa um mundo que mais se afigura àquele transmitido pelas emissoras (Rangel, 2004). É a influência de muitas horas de exposição a tal meio de comunicação de massa na formação de atitudes socialmente difundidas, como um processo de homogeneização das opiniões (Bryant \& Miron, 2004). Neste caso, trata-se de opiniões públicas

\footnotetext{
1 Uma resposta da crítica de Hughes (1980) pode ser encontrada em Gerbner et al. (1980a) e Gerbner, Gross, Morgan e Signorielli (1981) responderam a crítica de Hirsch (1980) e Hughes (1980).
} 
ou atitudes sociais, pois são compartilhadas amplamente na sociedade. E, neste sentido, os meios de comunicação de massa, particularmente a TV, têm uma forte influência na formação de tais atitudes (Oskamp \& Schultz, 2005).

Com o intuito de verificar estudos prévios realizados no contexto brasileiro, entrou-se com a expressão medo do crime no índice de assuntos do Periódicos Eletrônicos de Psicologia (PePSIC) e no Scientific Electronic Library Online (SciELO Brazil) mas não se encontrou qualquer artigo.

Assim, decidiu-se realizar o presente estudo por meio da internet, cujo objetivo é fazer um levantamento de quanto medo as pessoas sentem de serem vítimas de crimes violentos, quais as medidas de segurança que usam e correlações com a exposição à televisão. Parte-se da hipótese de que quanto mais horas o respondente afirmar dispender assistindo à televisão, mais medo do crime terá (horas de $\mathrm{TV} \rightarrow$ medo do crime [teoria do cultivo]). Além disso, acredita-se que não apenas os que mais assistem a TV apresentarão mais medo do crime, mas também a audiência do sexo feminino e com mais idade (hipótese da vulnerabilidade). Em acréscimo, tendo em vista a importância de variáveis sócio-demográficas para se entender os efeitos do cultivo, busca-se verificar se as horas de TV predizem o medo do crime controlando-se o nível de escolaridade e religiosidade, estado civil e classe sócio-econômica percebida dos participantes. Isso permitirá conhecer se os efeitos de cultivação são diferentes de acordo com esses grupos, verificando se as variáveis são fatores constituintes dos efeitos do cultivo, tratando especificamente dos efeitos no medo do crime (Gerbner \& Gross, 1976; Gerbner et al., 1980a).

Para se testar tais hipóteses, acima explicitadas, elaborou-se o presente estudo empírico de caráter correlacional (cross-sectional survey, Babbie, 1990) realizado pela internet. Outra variável a propósito que também pode ser importante para se entender os efeitos do cultivo é o tipo de survey utilizado (Doob \& Mcdonald, 1979). Neste sentido, este autor comentou que as relações encontradas no estudo de Gerbner e Gross (1974 citado por Doob \& Mcdonald, 1979) foram pequenas por eles terem feito uso do survey por telefone. Por outro lado, tem-se verificado maiores efeitos de cultivo para diversas variáveis dependentes pelo survey por telefone quando comparado ao realizado por correio ou entrevistas pessoalmente (Shrum, 2004, 2007). Escolheu-se realizar um survey pela internet, que tem apresentado razoáveis taxas de retorno em diversos estudos (de 39,6 a 34,6\%, ver meta-análise de Cook, Heath \& Thompson, 2000). Além disso, os participantes poderão responder às perguntas sobre o medo do crime em suas residências e sem a presença do pesquisador. Portanto, confiamos em obter respostas sinceras e espontâneas para o conjunto de itens do questionário elaborado pelo meio de coleta adotado certamente diminuir as chances de produzir constrangimento nos respondentes.

\section{Método}

Participantes

Os respondentes desta pesquisa compreendem usuários de e-mail e participantes de comunidades virtuais do Distrito Federal. Inicialmente, com uma lisa de e-mails em mãos foram submetidos e-mails convidando para a participação na pesquisa. Seguiu-se a orientação da literatura especializada de enviar convites concisos com uma indicação clara da instituição e dos responsáveis pela pesquisa, disponibilizando-se e-mails para contato (Manfreda \& Vehovar, 2008).

Além disso, como recomendam estes autores, tomaram-se os cuidados éticos da pesquisa off-line, salvaguardando a confidencialidade e anonimato, participação voluntária e evitando o constrangimento. Foram enviados mais de 2 mil e-mails, mas muitos retornaram (75 mensagens tipo Mail Delivery System, razão do problema: A entrega expirou [Mensagem muito velha]: endereço do destinatário rejeitado) mostrando que não estavam atualizados.

Um primeiro envio de questionários foi realizado de 16 de novembro de 2010 até 4 de janeiro de 2011, totalizando-se 891 e-mails enviados e 26 respostas, sendo uma taxa de $2,91 \%$ em 50 dias. Outro envio foi feito entre 3 de janeiro de 2010 e 10 de janeiro de 2011, totalizando 2114 e-mails enviados e 124 respostas, sendo uma taxa de 6,19\% em 8 dias. A amostra final se constituiu de um total de 150 participantes, sendo a maioria do sexo feminino (54\%), com média de idade de 32 anos ( $\mathrm{DP}=11,31$, Moda = 30 anos, sendo $90 \%$ da amostra formada por pessoas de 14 a 49 anos), residentes no Distrito Federal, principalmente em Brasília (52\%), de classe média (51,3\%), solteiros (50\%), pós-graduados (56\%) e se consideraram algo religiosos (33\%). 


\section{Procedimento}

0 procedimento inicial consistiu em coletar e-mails em comunidades virtuais do Distrito Federal, integrantes do site de relacionamento social Orkut e também utilizar e-mails de uma lista previamente utilizada em pesquisas prévias. Com esta lista em mãos, foram enviados e-mails com o link da versão online da Escala de Medo do Crime por meio da ferramenta Survey Monkey.

\section{Instrumento}

Escala do Medo do Crime. Buscou-se uma medida para aferir o medo do crime, que fosse breve e com um formato de resposta fácil, para que não desencorajassem os respondentes num web survey (Dillman, 2008). Esta medida (Fear of Crime Scale) foi apresentada no compêndio de medidas relacionadas com comportamentos e atitudes antissociais de Dahlberg, Toal, Swahn e Behrens (2005) e desenvolvida por Gorman-Smith et al. (2000) no contexto norte-americano. Portanto, uma versão em português desta medida foi utilizada no presente estudo para acessar o medo do crime no contexto brasileiro. A escala objetiva quantificar o medo subjetivo de ser vítima de crime violento em casa ou na vizinhança a partir de 10 itens divididos nos fatores Medo do Crime (itens 1, 2, 3 e 4) ancorados em escala de resposta de 4 pontos (sendo $0=$ Nenhum Medo, 1 = Um Pouco de Medo, 2 = Algum Medo e 3 = Muito Medo) e Medidas tomadas para enfrentar o crime $(5,6,7,8$, 9 e 10) respondidos numa escala dicotômica (Não $=0$ e Sim $=1$ ).

O primeiro fator, Medo do Crime, é formado por itens que buscam saber se o respondente tem medo de ser atacado ou roubado em casa ou apartamento, nas ruas do próprio bairro durante o dia, quando sai sozinho à noite no próprio bairro e quando sai com outras pessoas à noite no próprio bairro. Itens do segundo fator, Medidas tomadas para enfrentar o crime, buscam saber se o respondente, em função do medo, limita os lugares e vezes que faz compras, vai ao trabalho ou escola, que vai por conta própria, se compra arma para autodefesa, se usa sistema de segurança em casa e se muda para morar em outro lugar. A escala foi utilizada inicialmente por Gorman-Smith et al. (2000) em jovens de escolas públicas de Chicago, mas também com adultos e apresentou bons coeficientes de consistência interna para
Medo do crime $(\alpha=0,86)$ e Medidas tomadas para enfrentar o medo do crime $(\alpha=0,77)$.

No presente estudo também foram verificados índices de precisão adequados para o Medo do crime $(\alpha=0,82)$ e Medidas tomadas para enfrentar o medo do crime (K-R $20=0,62$ ). Três itens desta medida (11-13) objetivavam saber se os participantes possuíam armas de fogo, mas foram retirados desta versão em português deste questionário, ficando agora com 10 itens. Ao término do questionário, são abordadas questões de caráter sociodemográfico, como sexo e idade. Busca-se também por um item aferir quantas horas de televisão o participante assiste por semana. A Escala de Medo do Crime foi traduzida para o português pelo autor deste artigo. Buscou-se, portanto, conseguir a clareza e elegância na redação dos itens traduzidos, de modo a maximizar a participação dos respondentes. Testou-se a versão online em português da Escala de Medo do Crime com a participação de seis estudantes de variados níveis (ensino médio, graduação, pós-graduação) com o objetivo de verificar se esta medida é compreensível e se é convidativa para a participação. Além disso, programou-se esta medida para que não tivéssemos respostas missing. Portanto, os sujeitos teriam que responder a todos os itens. Os estudantes responderam a versão online da escala por meio de solicitações feitas pelo autor no MSN. Informou-se do objetivo geral da pesquisa e da importância de se testar uma versão prévia do questionário para a coleta de dados numa amostra maior. De acordo com a colaboração desses voluntários, não foram demandadas grandes alterações na medida em questão, considerando-a uma medida de fácil utilização e compreensão, concisa e com uma interface amigável (Manfreda \& Vehovar, 2008).

\section{Análise dos dados}

A análise dos dados compreende uma análise estatística, considerando estatística descritiva (média [M], desvio padrão [DP], frequências [f] e percentual [\%]) e estatística inferencial (análise de correlação de Pearson, Spearman, correlação parcial, regressão simples e múltipla). Também foram realizadas análises gráficas por meio dos scatter plots. Todos os dados foram analisados no programa estatístico SPSS (Statistical Package for Social Sciences) para Windows ${ }^{\circledR}$ versão 15. 


\section{Resultados}

Inicialmente realizaram-se análises descritivas com o fim de verificar o grau de medo do crime que os participantes indicaram. Verificou-se que $40,7 \%$ dos participantes têm um pouco de medo (ponto 1 da escala) de ser atacado ou roubado em casa ou no apartamento e que $40,7 \%$ não têm nenhum medo de ser atacado ou roubado neste contexto. Com relação a ser atacado ou roubado nas ruas do seu bairro durante o dia, $44 \%$ afirmaram ter um pouco de medo. Quanto ao medo de ser roubado ou atacado quando sai sozinho durante a noite, no seu bairro, $42 \%$ indicaram algum medo (ponto 2). Em resposta à pergunta se tem medo de ser atacado ou roubado quando sai com outras pessoas no seu bairro, $36 \%$ indicaram um pouco de medo e $32 \%$ algum medo.

Tendo em conta agora as medidas tomadas para enfrentar o medo do crime, verificou-se que $55,7 \%$ indicaram que ter medo do crime não os faz limitar os lugares que vão para fazer compras. Um total de $76,4 \%$ não limitam os lugares e às vezes que vão ao trabalho ou escola; cerca de $61 \%$ limitam os lugares que vão por conta própria; mais de $90 \%$ não compram uma arma para se proteger; $50 \%$ não instalam sistema de segurança ou dispositivos de proteção como grades nas janelas, campainhas em janelas e/ou portas em casa. Por fim, cerca de $72 \%$ não mudam para morar em outro lugar.

\section{Medo do crime, horas assistindo à TV por semana, sexo e idade}

Com o fim de responder à questão principal da presente pesquisa, verificou-se a média de medo do crime segundo os participantes desta pesquisa e correlacionou-se a medida de medo do crime com o total das horas que os participantes afirmaram assistir a TV por semana. Considerando o fator de Medo do Crime, verificou-se que em média os participantes afirmaram ter um pouco de medo do crime $(\mathrm{M}=1,23, \mathrm{DP}=0,70)$. Considerando o fator de Medidas tomadas para enfrentar o medo do crime, verificou-se que $62 \%$ não limitam os lugares ou vezes que vai às compras, trabalho, por conta própria, não compra armas para se proteger, nem instala sistema de segurança em casa nem muda de moradia para outro lugar.

Em média, estes participantes passam 9,67 horas vendo TV por semana. A correlação de Pearson mostrou que quanto mais horas de TV os participantes afirmaram assistir por semana, mais medo do crime reportaram ter $(r=0,23, p<0,005)$. Na mesma direção, mas mais sutilmente, verificou-se correlação entre horas de TV e medidas tomadas para enfrentar o medo do crime $(r=0,15, p<0,05)$. Controlando-se a idade numa correlação parcial estas correlações não se alteraram. Tais fatores, medo do crime e medidas tomadas para enfrentar o crime, se mostraram positivamente correlacionados $(r=0,52, p<0,001)$. Não se verificou, por outro lado, qualquer correlação entre medo do crime ou medidas tomadas para enfrentar o crime, sexo e idade.

Porém considerando apenas a amostra masculina, não se verificaram correlações entre medo do crime e horas de TV, mas esta relação se verificou na amostra feminina $(r=0,29, p<0,005)$. Nenhuma correlação foi verificada com estas amostras tendo em vista as medidas para enfrentar o medo do crime e as horas de TV. Verificou-se ainda por meio de análise de regressão simples que as horas de TV predizem o medo do crime $\left(R=0,24, R^{2}=0,05, F=8,400, p<0,005\right)$, explicando cerca de $5 \%$ da variância do medo do crime. No entanto, conforme se observa na Gráfico 1, a análise dos scatter plot mostra que esta relação é melhor entendida como cúbica ao invés de linear.

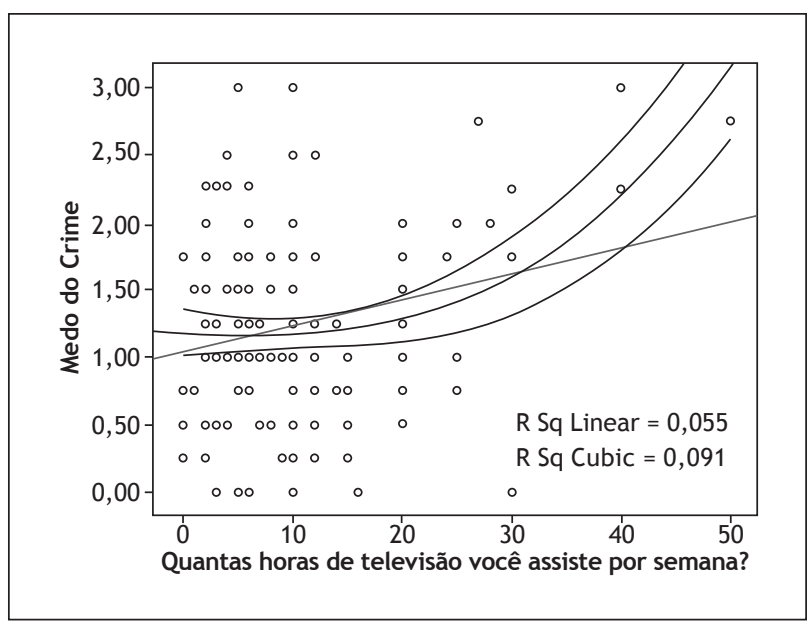

Gráfico 1 - Scatter plot da relação entre horas de TV e medo do crime Fonte: Dados da pesquisa.

Outro controle foi tomado no que diz respeito à identificação de respondentes que apresentaram uma elevada quantidade de horas de TV por semana. Encontraram-se, pelo valor padronizado desta variável (z), três respondentes que podem ser considerados 
desviantes (outliers): sendo um reportando 50 horas semanais e dois afirmando assistir a 40 horas semanais de TV, como se pode ver na figura anterior. Tais respondentes tornaram a relação cúbica. A exclusão desses participantes diminui a média para 8,98 e torna não estatisticamente significativa as correlações (lineares) entre horas de TV e os fatores de medo do crime.

Considerando agora apenas aqueles com um mínimo de 14 horas de TV por semana $(n=30)$, verificamos correlações (Pearson e Spearman) ainda maiores com medo do crime $(r=0,49, p<0,005, \rho=0,48, p<0,005)$ como se observa no Gráfico 2 a seguir. A correlação se mantém mesmo se controlando a idade $(\mathrm{r}=0,44, \mathrm{p}$ $<0,05)$ ou o sexo $(r=0,49, \mathrm{p}<0,005)$ ou a idade e o sexo conjuntamente $(r=0,45, p<0,01)$. Mas não se verificou nenhuma correlação com medidas tomadas para enfrentar o crime. Em média estes telespectadores passaram 20 horas assistindo à TV por semana (Gráfico 2).

Por fim, as horas de TV predisseram o medo do crime $(\beta=0,49, p<0,007)$, mesmo ao se controlar a idade, $o$ sexo, a classe socioeconômica percebida, o nível de escolaridade, estado civil e religiosidade $(\beta=0,45, p<0,05){ }^{2}$

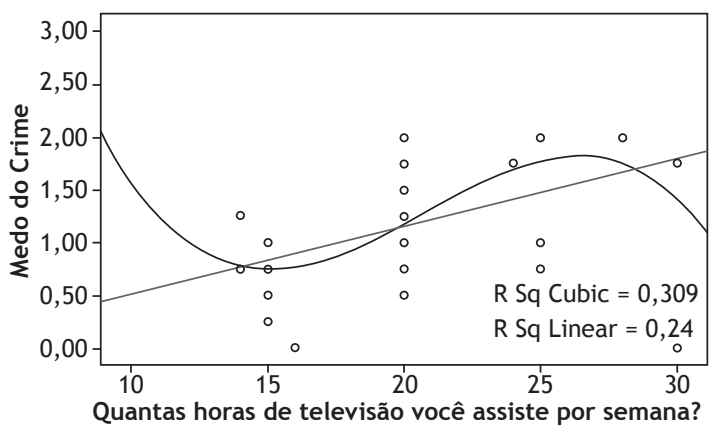

Gráfico 2 - Scatter plot da relação entre horas de TV e medo do crime e entre heavy viewers

Fonte: Dados da pesquisa.

Enfim, mesmo que as relações entre medo do crime e horas de TV possam ser explicadas satisfatoriamente de modo linear, uma vez mais tendo em conta a análise gráfica, o modelo cúbico se mostrou mais adequado. Verificou-se que ocorreu um aumento do medo do crime nos que assistiam a mais horas de TV, mas que também houve um decréscimo desse medo.

\section{Discussão}

Nesta pesquisa, por meio de um survey pela internet, testou-se a hipótese de que as horas de TV predizem o medo do crime. Testou-se também a hipótese da vulnerabilidade, segundo a qual a audiência do sexo feminino e com mais idade apresentará mais medo do crime. Verificou-se ainda a viabilidade do levantamento de dados por meio da web para as questões pesquisadas. Deve-se destacar, por outro lado, o baixo percentual de retorno dos e-mails enviados (menos de 10\%) em relação a outros surveys realizados pela internet (Cook et al., 2000). Isto talvez seja um reflexo de segmentos do nosso contexto cultural que não tem apoiado tanto a pesquisa científica em termos de disponibilidade para responder questionários. Torna-se, portanto, um desafio para o pesquisador realizar pesquisas com grandes amostras.

Com base nos principais resultados do presente estudo, avaliamos que os dados ora apresentados corroboram a primeira hipótese, oriunda da teoria do cultivo (Gerbner \& Gross, 1976; Gerbner et al., 1980a, b) e outros estudos prévios (Bulck, 2004; Doob \& Mcdonald, 1979; Holbert, Shah \& Kwak, 2004; O’Keefe \& Reid-Nash, 1987; Romer, Jamieson \& Aday, 2003) mas não confirmam a hipótese da vulnerabilidade (Barnett et al., 2007; McCrea et al., 2005; Fetchenhauer \& Buunk, 2005). Deve-se salientar, por outro lado, que a grande maioria dos respondentes dessa pesquisa é de jovens e adultos internautas, mas não de idosos. 0 que sugere que a hipótese da vulnerabilidade não ocorre na comparação desses grupos.

Mesmo verificando que ver TV predisse o medo do crime, deve-se ponderar que os participantes desta pesquisa reportaram apenas um pouco de medo. Os resultados da presente pesquisa mostraram que as relações entre horas de TV e medo só se confirmam naqueles telespectadores que assistem a muitas horas de TV (Gerbner \& Gross, 1976) o que representou $20 \%$ da amostra total. No estudo original de Gerbner e Gross, foram considerados heavy viewers aqueles que passavam em média 21 horas semanais vendo TV. Encontramos tamanho de efeito médio entre medo e horas de TV entre aqueles que passavam em média 20 horas semanais vendo TV, 2 As variáveis categóricas, classe socioeconômica, estado civil e nível de escolaridade foram transformadas em
variáveis dummy para entrarem na equação de regressão como variáveis de controle. 
controlando-se o sexo, a idade, o nível de escolaridade e religiosidade, estado civil e classe socioeconômica percebida dos participantes. Não se verificou, por outro lado, qualquer correlação com medidas tomadas para enfrentar o crime. Tais coeficientes são maiores do que aqueles encontrados em estudos prévios sobre efeitos do cultivo (Doob \& Mcdonald, 1979; Holbert et al., 2004; Morgan \& Shanahan, 1996).

Em suma, assistir à TV predisse medo do crime, mas não se pode falar em relações de causa e efeito nesta pesquisa por sua natureza correlacional ou não experimental (Shadish, Cook \& Campbell, 2002). Deste modo, pode-se especular que o medo do crime leva as pessoas a ficarem mais tempo em casa o que consequentemente oportunizará maior tempo para assistir à programação televisiva (withdrawal hypothesis, Bulck, 2004; Wåhlberg \& Sjöberg, 2000). Mas também que as pessoas veem mais TV e sentem mais medo do crime (teoria do cultivo) ou que estas relações são melhor explicadas por um terceiro fator desconhecido.

A despeito desta limitação metodológica, isto é, não se poder falar em relação de causa e efeito, ressalte-se que se optou por este delineamento no presente estudo com o fim de verificar as variáveis em apreço na sua manifestação natural. Não entrando no conflito ético de se manipular variáveis com o fim de se provocar medo nos participantes da pesquisa. Nestes termos, talvez o delineamento mais adequado para se verificarem os efeitos do cultivo seja um delineamento longitudinal (Ruspini, 2002). Finalmente, outro ponto importante de destacar é que as relações encontradas talvez sejam melhor entendidas a partir de um modelo não linear de fato (Hirsch, 1980), do tipo cúbico. E, finalmente, não se pretende generalizar os resultados uma vez que se tratou com uma amostra não probabilística.

A relação não linear mostrada nos scatter plots significa que o medo do crime até caiu entre alguns heavy viewers, o que pode ser entendido como efeitos de desensibilização (Carnagey, Anderson \& Bushman, 2007). Ou seja, a exposição repetida à violência na mídia, como programas televisivos, pode levar a um decréscimo nas emoções relacionadas (Cline, Croft \& Courrier, 1973; Krahé, Möller, Huesmann, Kirwil, Felber \& Berger, 2010; Mullin \& Linz, 1995; Thomas, Horton, Lippincott \& Drabman, 1977). Mas esta hipótese não foi testada no presente estudo, o que vem sendo feito de modo experimental, a exemplo das pesquisas supracitadas.
Aponta-se como uma limitação do estudo o fato de não se terem controlado as relações averiguadas com base em outras variáveis. Tendo isto em apreço, pode-se futuramente pesquisar, com o tempo de exposição, quais programas na TV predizem mais medo do crime comparando-se tipos de survey distintos, como pela internet, telefone e carta (Shrum, 2004; 2007). Nesta pesquisa levou-se em conta a dimensão subjetiva deste medo, mas indicadores fisiológicos também podem ser investigados.

A TV de fato é um veículo de comunicação privilegiada quando se pensa nos seus efeitos sociais e psicológicos e há décadas tem chamado a atenção dos psicólogos sociais (DeFleur \& Ball-Rokeach, 1993; Johnson, Maio \& Smith-McLallen, 2005; Weiss, 1968). Tendo em conta especificamente o medo do crime, novas pesquisas devem ser realizadas em contextos variáveis em função da quantidade de delitos (bairros mais e menos violentos) e considerando amostras maiores de telespectadores mais assíduos. Estes e outros intuitos devem ser levados em conta quando se pretender replicar as relações ora apresentadas, contribuindo de modo mais abrangente e preciso para se compreender os efeitos do cultivo.

\section{Referências}

Babbie, E. (1990). Survey research methods. New York: Wadsworth.

Barnett, K., Buys, L., Lovie-Kitchin, J., Boulton-Lewis, G., Smith, D., \& BBus, M. H. (2007). Older women's fears of violence: The need for interventions that enable active ageing. Journal of Women \& Aging, 19 (3/4), 179-193.

Black Sabbath. (1992). TV Crimes. On Dehumanizer [CD]. New York: Reprise Records.

Bryant, J., \& Miron, D. (2004). Theory and research in mass communication. Journal of Communication, 54 (4), 662-704.

Bulck, J. V. den. (2004). Research note: The relationship between television fiction and fear of crime: An empirical comparison of three causal explanations. European Journal of Communication, 19(2), 239-248.

Bushman, B. J., \& Anderson, C. (2001). Media violence and the American public: Scientific facts versus media misinformation. American Psychologist, 56(6/7), 477-489. 
Carnagey, N. L., Anderson, C.A., \& Bushman, B. J. (2007). The effect of video game violence on physiological desensitization to real-life violence. Journal of Experimental Social Psychology, 43(3), 489-496.

Cline, V. B., Croft, R. G., \& Courrier, S. (1973). Desensitization of children to television violence. Journal of Personality and Social Psychology, 27(3), 360-365.

Cook, L. L., Heath, F., \& Thompson, R. L. (2000). A meta-analysis of response rates in web- or Internet-based surveys. Educational and Psychological Measurement, 60(6), 821-836.

Dahlberg, L. L., Toal, S. B., Swahn, M., \& Behrens, C. B. (2005). Measuring violence-related attitudes, behaviors, and influences among youths: A compendium of assessment tools. Atlanta: Centers for Disease Control and Prevention, National Center for Injury Prevention and Control.

DeFleur, M. L., \& Ball-Rokeach, S. (1993). Teorias da comunicação de massa. Rio de Janeiro: J. Zahar.

Dillman, D. (2008). The logic and psychology of constructing questionnaires. In E. D. de Leeuw, J. L. Hox, \& D. A. Dillman (Ed.). International handbook of survey methodology (pp. 161-175). New York: Psychology Press.

Doob, A. N., \& McDonald, G. E. (1979). Television viewing and fear of victimization: Is the relationship causal? Journal of Personality and Social Psychology, 37(2), 170-179.

Dowler, K. (2003). Media consumption and public attitudes toward crime and justice: The relationship between fear of crime, punitive attitudes, and perceived police effectiveness. Journal of Criminal Justice and Popular Culture, 10(2), 109-126.

Fetchenhauer, D., \& Buunk, B. P. (2005). How to explain gender differences in fear of crime: Towards an evolutionary approach. Sexualities, Evolution and Gender, 7(2), 95-113.

Gerbner, G., (1970). Cultural indicators: The case of violence in television drama. Annals of the American Academy of Political and Social Science, 388(1), 69-81.

Gerbner, G., \& Gross, L. (1976). Living with television: The violence profile. Journal of Communication, 26(2), 172-199.
Gerbner, G., Gross, L., Morgan, M., \& Signorielli, N. (1979). On Wober's televised violence and paranoid perception: The view from great britain. The Public Opinion Quarterly, 43(1), 123-124.

Gerbner, G., Gross, L., Morgan, M., \& Signorielli, N. (1980a). Additional comments on cultivation analysis. The Public Opinion Quarterly, 44(3), 408-410.

Gerbner, G., Gross, L., Morgan, M., \& Signorielli, N. (1980b). The "mainstreaming" of America: Violence profile $\mathrm{n}$ 은 11. Journal of Communication, 30(3), 10-29.

Gerbner, G., Gross, L., Morgan, M., \& Signorielli, N. (1981). On the limits of "The limits of advocacy research": Response to Hirsch. The Public Opinion Quarterly, 45(1), 116-118.

Gerbner, G., Gross, L., Morgan, M., \& Signorielli, N. (1984). Political correlates of television viewing. The Public Opinion Quarterly, 48(1), 283-300.

Gerbner, G., Gross, I., Morgan, M., Signorielli, N., \& Shanahan, J. (2002). Growing up with television: Cultivation processes. In J. Bryant \& D. Zillmunn (Ed.). Media effects: Advance in theory and research (pp. 43-67). Mahwah: Lawrence Erlbuum Associates.

Gorman-Smith, D., Tolan, P. H., \& Henry, D. B. (2000). A developmental-ecological model of the relation of family functioning to patterns of delinquency. Journal of Quantitative Criminology, 16(2), 169-198.

Gunter, B., \& Harrison, J. (2005). Violence and television: An analysis of amount, nature, location and origin of violence in British programmes. New York: Routledge Progress in Psychology.

Hirsch, P. M. (1980). On Hughes's contribution: The limits of advocacy research. The Public Opinion Quarterly, 44(3), 411-413.

Holbert, R. L., Shah, D. V., \& Kwak, N. (2004). Fear, authority, and justice: Crime-related TV viewing and endorsements of capital punishment and gun ownership. J\&MC Quarterly, 81(2), 343-363.

Hughes, M. (1980). The fruits of cultivation analysis: A reexamination of some effects of television watching. The Public Opinion Quarterly, 44(3), 287-302. 
Johnson, B. T., Maio, G. R., \& Smith-McLallen, A. (2005). Communication and attitude change: Causes, processes, and effects. In D. Albarracín, B. T. Johnson \& M. P. Zanna (Ed.). The handbook of attitudes (pp. 617-670). London: Lawrence Erlbaum Associates.

Krahé, B., Möller, I., Huesmann, L. R., Kirwil, L., Felber, J., \& Berger, A. (2010). Desensitization to media violence: Links with habitual media violence exposure, aggressive cognitions, and aggressive behavior. Journal of Personality and Social Psychology, 100(4), 630646. doi: 10.1037/a0021711

Manfreda, K. L., \& Vehovar, V. (2008). Internet surveys. In E. D. de Leeuw, J. J. Hox \& D. A. Dillman (Ed.). International handbook of survey methodology (pp. 264284). New York: Psychology Press.

McCrea, R., Shyy, T-K., Western, J., \& Stimson, R. J. (2005). Fear of crime in Brisbane: Individual, social and neighbourhood factors in perspective. Journal of Sociology, 41(7), 7-27.

Mora, M. N. (2009). El miedo al delito no es un supuesto de victimización indirecta. International e-Journal of Criminal Science, 3(3), 1-40.

Morgan M., \& Shanahan J. (1996). Two decades or cultivation research: An appraisal and meta-analysis. In B. R. Burleson (Ed.). Communication Yearbook 20 (pp. 1-45). Newbury Park: Sage.

Mullin, C. R., \& Linz, D. (1995). Desensitization and resensitization to violence against women: Effects of exposure to sexually violent films on judgments of domestic violence victims. Journal of Personality and Social Psychology, 69(3), 449-459.

Niederdeppe, J., Fowler, E. F., Goldstein, K., \& Pribble, J. (2010). Does local television news coverage cultivate fatalistic beliefs about cancer prevention? Journal of Communication, 60(1), 230-253.

O’Keefe, G. J., \& Reid-Nash, K. (1987). Crime news and real-world blues: The effects of the media on social reality. Communication Research, 14(2), 147-163.

Oskamp, S., \& Schultz, P. W. (2004). Attitudes and opinions. London: Lawrence Erlbaum Associates.

Rangel, J. G. (2004). Televisão em foco: A violência e suas relações psicossociais no contexto dos telespectadores. ALCEU, 4(8), 51-64.
Romer, D., Jamieson, K. H., \& Aday, S. (2003). Television news and the cultivation of fear of crime. Journal of Communication, 53(1), 88-104.

Ruspini, E. (2002). Introduction to longitudinal research. London: Routledge.

Saito, S. (2007). Television and the cultivation of gender-role attitudes in Japan: Does television contribute to the maintenance of the status quo? Journal of Communication, 57(3), 511-531.

Shadish, W., Cook, T., \& Campbell, D. (2002). Experimental \& quasi-experimental designs for generalized causal inference. Boston: Houghton Mifflin.

Shrum, L. J., Burroughs, J. E., \& Rindfleisch, A. (2004). A process model of consumer cultivation: The role of T.V. is a function of type of judgment. In L. J. Shrum (Ed.). The psychology of entertainment media: Blurring the lines between entertainment and persuasion (pp. 177-192). Mahwah: Lawrene Erlbuum Associates.

Shrum, L. J. (2004). Magnitude of effects of television viewing on social perceptions vary as a function of data collection method: Implications for psychological processes. Advances in Consumer Research, 31, 511-513.

Shrum, L. J. (2007). The implications of survey method for measuring cultivation effects. Human Communication Research, 33(1), 64-80.

Thomas, M. H., Horton, R. W., Lippincott, E. C., \& Drabman, R. S. (1977). Desensitization to portrayals of real life aggression as a function of television violence. Journal of Personality and Social Psychology, 35(6), 450-458.

Wåhlberg, A. A., \& Sjöberg, L. (2000). Risk perception and the media. Journal of Risk Research, 3(1), 31-50.

Waiselfisz, J. J. (2010). Mapa da violência: Anatomia dos homicídios no Brasil. São Paulo: Instituto Sangari.

Weiss, W. (1968). Effects of the mass media of communication. In: G. Lindzey \& E. Aronson (Eds.). The handbook of social psychology (pp. 77-196). California: Addison-Wesley Publishing Company.

Williams, D. (2006). Virtual cultivation: Online worlds, offline perceptions. Journal of Communication, 56(1), 69-87. 
